\title{
The mPEG-PCL Copolymer for Selective Fermentation of Staphylococcus lugdunensis Against Candida parapsilosis in the Human Microbiome
}

Ming-Shan Kao', Yanhan Wang ${ }^{2}$, Shinta Marito', Stephen Huang ${ }^{3}$, Wan-Zhen Lin ${ }^{1}$, Jon A Gangoiti ${ }^{4}$, Bruce A Barshop ${ }^{4}$, Choi Hyun ${ }^{4}$, Woan-Ruah Lee ${ }^{5}$, James A Sanford ${ }^{2}$, Richard L Gallo ${ }^{2}$, Yuping Ran ${ }^{6}$, Wan-Tzu Chen ${ }^{7}$, Chun-Jen Huang ${ }^{8}$, Ming-Fa Hsieh ${ }^{7 *}$ and Chun-Ming Huang ${ }^{2,9 *}$

${ }^{1}$ Department of Life Sciences, National Central University, Taoyuan, Taiwan

${ }^{2}$ Department of Dermatology, University of California, San Diego, CA, USA

${ }^{3}$ Surface Bioadvances Inc., San Diego, CA, USA

${ }^{4}$ Department of Pediatrics University of California, San Diego, CA, USA

${ }^{5}$ Department of Dermatology, Taipei Medical University, Taipei, Taiwan

${ }^{6}$ Department of Dermatology, West China Hospital, Sichuan University, Chengdu, China

${ }^{7}$ Department of Biomedical Engineering, Chung Yuan Christian University, Taoyuan, Taiwan

${ }^{8}$ Department of Biomedical Sciences and Engineering, National Central University, Taoyuan, Taiwan

${ }^{9}$ Moores Cancer Center; University of California, San Diego, CA, USA

\begin{abstract}
Many human skin diseases, such as seborrheic dermatitis, potentially occur due to the over-growth of fungi. It remains a challenge to develop fungicides with a lower risk of generating resistant fungi and non-specifically killing commensal microbes. Our probiotic approaches using a selective fermentation initiator of skin commensal bacteria, fermentation metabolites or their derivatives provide novel therapeutics to rein in the over-growth of fungi. Staphylococcus lugdunensis (S. Iugdunensis) bacteria and Candida parapsilosis (C. parapsilosis) fungi coexist in the scalp microbiome. S. lugdunensis interfered with the growth of $C$. parapsilosis via fermentation. A methoxy poly (ethylene glycol)- $b$-poly ( $\varepsilon$-caprolactone) (mPEG-PCL) copolymer functioned as a selective fermentation initiator of $S$. Iugdunensis, selectively triggering the $S$. Iugdunensis fermentation to produce acetic and isovaleric acids. The acetic acid and its pro-drug diethyleneglycol diacetate (Ac-DEG-Ac) effectively suppressed the growth of $C$. parapsilosis in vitro and impeded the fungal expansion in the human dandruff. We demonstrate for the first time that $S$. lugdunensis is a skin probiotic bacterium that can exploit $\mathrm{mPEG}-\mathrm{PCL}$ to yield fungicidal short-chain fatty acids (SCFAs). The concept of bacterial fermentation as a part of skin immunity to re-balance the dysbiotic microbiome warrants a novel avenue for studying the probiotic function of the skin microbiome in promoting health.
\end{abstract}

Keywords: C. parapsilosis; Fermentation; Microbiome; Probiotic; $S$. lugdunensis

\section{Introduction}

The skin is colonized by a diverse array of microorganisms including bacteria and fungi. The skin microbiome is defined as the collection of all microbes that colonize the skin [1]. Environments at various topographical areas of skin can affect the microbial colonization. The major bacterial-fungal populations colonizing human scalps have been characterized [2,3]. Furthermore, the dysbiosis of bacterial-fungal populations has been implicated in scalp dandruff, which presents as significant problems to large numbers of people [4]. Dandruff scalps are associated with a higher abundance of Malassezia restricta and Staphylococcal species [5]. The severity of dandruff can range from mild scale formation similar to dry skin to seborrheic dermatitis [6]. The impairment of proper hydration in skin barrier can result in the typical epidermal proliferation, keratinocyte differentiation and stratum corneum maturation, which may cause dandruff [7]. Excessive secretion of the sebaceous gland also can underlie dandruff development. Besides dysfunction of the skin barrier and sebaceous gland, fungal/bacterial dysbiosis may be one of the factors that result in the progression of human dandruff.

Bacterial interference, or bacteriotherapy, in which commensal bacteria as probiotics are used to rein in the over-growth of opportunistic microbes, has been shown to be a promising modality for normalization of dysbiosis in the human microbiome [8-14]. Our pioneer studies demonstrated that the skin commensal bacteria can function as probiotic bacteria to undergo fermentation and produce short-chain fatty acids (SCFAs) [15]. Staphylococcus epidermidis (S. epidermidis), a skin commensal bacterium, can exploit glycerol fermentation to restrain the over-growth of opportunistic Propionibacterium acnes ( $P$. acnes) [16]. Succinic acid, one of the SCFAs in fermentation products of S. epidermidis, inhibits the P. acnes growth in vitro and in vivo. Based on the data from our previous studies, we conjecture that production of SCFAs by probiotic bacteria in skin is a part of innate immunity to equilibrate the dysbiosis of the skin microbiome. In fact, it has been documented that SCFAs, although concentrations are relatively low in the skin, played a crucial role in altering the predominant residence of bacteria on normal human skin [17]. Many SCFAs with potent antimicrobial activities have been approved by the U.S. Environmental Protection Agency (EPA) as active ingredients for use as fungicides and bactericides on stored grains, poultry litter, and drinking water for poultry and livestock $[18,19]$. Furthermore, the Food and Drug Administration (FDA) has approved many SCFAs as flavor enhancers, miscellaneous and general purpose food chemicals, neutralizing agents, and $\mathrm{pH}$ control agents [20].

*Corresponding author: Chun-Ming Huang, Department of Dermatology, University of California, San Diego, CA, USA, Tel: 858-822-4627; E-mail chunming@ucsd.edu

Ming-Fa Hsieh, Department of Biomedical Engineering, Chung Yuan Christian University, Taoyuan, Taiwan, Tel: +886 3265 9999; E-mail: mfhsieh@cycu.edu.tw

Received May 12, 2016; Accepted June 09, 2016; Published June 19, 2016

Citation: Kao MS, Wang Y, Marito S, Huang S, Lin WZ, et al. (2016) The mPEGPCL Copolymer for Selective Fermentation of Staphylococcus lugdunensis Agains Candida parapsilosis in the Human Microbiome. J Microb Biochem Technol 8: 259 265. doi: 10.4172/1948-5948.1000295

Copyright: ( $) 2016$ Kao MS, et al. This is an open-access article distributed unde the terms of the Creative Commons Attribution License, which permits unrestricted use, distribution, and reproduction in any medium, provided the original author and source are credited. 
Citation: Kao MS, Wang Y, Marito S, Huang S, Lin WZ, et al. (2016) The mPEG-PCL Copolymer for Selective Fermentation of Staphylococcus lugdunensis Against Candida parapsilosis in the Human Microbiome. J Microb Biochem Technol 8: 259-265. doi: 10.4172/19485948.1000295

Currently, fungicides are the most effective drugs for treating dandruff. The zinc pyrithione (ZPT), selenium sulfide, coal tar and ketoconazole have been approved by the FDA to improve the dandruff by removal of fungi [7]. Keratolytic agents such as salicylic acid and sulfur were used to loosen the attachments between the corneocytes, allowing dandruff to get washed off [21]. However, the literature increasingly demonstrates that the prolong use of fungicides for treating a fungal infection of the scalp can be highly toxic [22]. The side-effects of keratolytic agents include dryness and irritation.

In the present study, we have identified and isolated Staphylococcus lugdunensis ( $S$. lugdunensis) bacterium and Candida parapsilosis (C. parapsilosis) fungus from human dandruff flakes. We also demonstrated that S. lugdunensis can counteract the growth of C. parapsilosis via fermentation, validating the probiotic activity of $S$. lugdunensis. It has reported that bacteria can use a polyethylene glycol (PEG) polymer as a carbon source and fermentatively convert PEG to acetate and ethanol [23]. A diblock methoxy poly(ethylene glycol)- $b$-poly( $\varepsilon$ caprolactone) (mPEG-PCL) polymer was synthesized as a selective fermentation initiator which can exclusively trigger the fermentation of $S$. lugdunensis, but not C. parapsilosis, to yield acetate. We also revealed that acetate and its analog exert excellent activities against the growth of fungi in human dandruff flakes.

A prevalence of dandruff of up to $50 \%$ was found in the general population and approximately 50 million people suffer from dandruff in the United States (U.S.) with nearly $\$ 300$ million spent on various dandruff treatment products annually [24]. Thus, the significance in this study includes providing a brand new approach to treat the fungal infection of the scalp skin, thereby benefiting the entire community of patients with dandruff or seborrheic dermatitis. Besides dandruff, hyperalimentation solutions, prosthetic devices, indwelling catheters and the nosocomial spread of disease through the hands of health care workers can be caused by C. parapsilosis infections [25]. Therefore, fermenting $S$. lugdunensis bacteria and their ferment metabolites may be novel therapeutics for the treatment of C. parapsilosis-associated infections.

\section{Materials and Methods}

\section{Dandruff collection and microbial growth}

Ethical approval for dandruff collection was obtained from Department of Dermatology, Taipei Medical University, Taiwan. The written consents from all participants were obtained before conducting dandruff collection. Those dandruff flakes with sizes greater than 1 $\mathrm{mm}^{2}$ were placed on malt extract agar (MEA) (Scharlab, S.L., Barcelona, Spain) plate's right after dandruff collection. Agar plates with dandruff flakes were incubated at $30^{\circ} \mathrm{C}$ until the microbial colonies were formed.

\section{Microbial identification}

Colonies on MEA plates were picked up by sterile toothpicks and DNA was extracted by an EasyPure Genomic DNA Spin kit (Bioman Scientific Co., Ltd, Taipei, Taiwan). For bacterial identification, polymerase chain reaction (PCR) with $16 \mathrm{~S}$ rRNA $27 \mathrm{~F}$ and $534 \mathrm{R}$ primers in addition to sequencing of PCR products was conducted as previously described [26]. For fungal identification, the D1/D2 5.8S rRNA gene was sequenced directly from the PCR products by using the primer pairs ITS1-Reverse (5'-TCCGTAGGTGAACCTGCGG-3') and ITS4-Forward (5'-TCCTCCGCTTATTGATATGC-3') [27]. PCR was performed under the following conditions: after an initial 3 min denaturation step at $95^{\circ} \mathrm{C}, 28$ cycles of amplification were performed, each including $30 \mathrm{sec}$ denaturation at $95^{\circ} \mathrm{C}, 30 \mathrm{sec}$ annealing at $52^{\circ} \mathrm{C}$ and $1.5 \mathrm{~min}$ extension at $72^{\circ} \mathrm{C}$, followed by a final 5 min extension at $72^{\circ} \mathrm{C}$. The gene sequences of both $16 \mathrm{~S}$ rRNA and D1/D2 5.8S rRNA were analyzed using the basic local alignment search tool (BLASTn).

\section{Culture of microbes}

Identified bacteria and fungi were cultured in tryptic soy broth (TSB) and potato dextrose agar (PDA) (Sigma, St. Louis, MO, USA), respectively. Overnight cultures were diluted 1:100 and cultured to an absorbance at $600 \mathrm{~nm}$ [optical density $\left.(\mathrm{OD})_{600}\right]=1.0$. For some experiments, microbes were harvested by centrifugation at $5,000 \mathrm{~g}$ for $10 \mathrm{~min}$, washed with phosphate buffered saline (PBS), and suspended in PBS.

\section{Co-culture of S. lugdunensis and C. parapsilosis}

S. lugdunensis $\left[10^{5}\right.$ colony forming unit (CFU)] was co-cultured with C. parapsilosis $\left(10^{5} \mathrm{CFU}\right)$ in rich media $(10 \mathrm{ml})[10 \mathrm{~g} / \mathrm{l}$ yeast extract (Biokar Diagnostics, Beauvais, France), $3 \mathrm{~g} / \mathrm{l} \mathrm{TSB}, 2.5 \mathrm{~g} / \mathrm{K} \mathrm{K}_{2} \mathrm{HPO}_{4}$ and $\left.1.5 \mathrm{~g} / \mathrm{l} \mathrm{KH}_{2} \mathrm{PO}_{4}\right]$ in the presence or absence $20 \mathrm{~g} / \mathrm{l}$ glycerol. After 3-day culture, media containing the microbes with a serial dilution $\left(1-10^{5}\right.$ CFU in $\left.10 \mu \mathrm{H}_{2} \mathrm{O}\right)$ were spotted on furazolidone $(10 \mu \mathrm{g} / \mathrm{ml}$; Sigma $)$ containing PDA plates for 3 days.

\section{Synthesis and characterizations of mPEG-PCL}

The mPEG-PCL diblock polymer was synthesized by ring-opening polymerization of e-caprolactone (Sigma). Monomer e-caprolactone (0.308 moles) was introduced in round-bottom flask along with macro initiator methoxy poly(ethylene glycol) [mPEG, molecular weight $(\mathrm{Mw}=550,3.96 \mathrm{mmoles})$ under purging of nitrogen gas. The mixture was then heated up. When the temperature reached $130^{\circ} \mathrm{C}$, the catalyst stannous 2-ethyl hexanoate (Sigma) $(0.272 \mathrm{mmoles})$ was added into the flask for $5 \mathrm{~h}$. The product was firstly dissolved in dichloromethane and then precipitated in ether/hexane of a volumetric ratio of 7:3 for three times. The precipitated samples were collected and vacuum dried. The $\mathrm{Mw}$ of the polymer was measured by gel permeation chromatography (GPCmax VE2001, Viscotek, Texas, USA) connected to a refractive index detector (VE3580, Viscotek, Texas, USA). Two columns (500 and 1000 angstroms, American Polymer Standards Corporation, USA) were maintained at $40^{\circ} \mathrm{C}$. The polystyrene standards ( $\mathrm{Mw}=972,6,480,9,000$, and 18,200, Polymer Standards Service GmBH, German) were used to create the calibration line for the determination of Mw. The mPEG-PCL polymer was characterized by Fourier-transform Infrared spectroscopy (FT-IR) (FT-IR 410, JASCO, Tokyo, Japan) for the functional groups in the molecular structure of the polymer. The powdery mPEG-PCL polymer was compressed into a potassium bromide $(\mathrm{KBr})$ plate for FT-IR measurements. The melting point which is related to Mw was measured by differential scanning calorimetry (DSC) (Jade DSC, Perkin-Elmer, Waltham, USA).

\section{Microbial fermentation}

S. lugdunensis and C. parapsilosis $\left(10^{5} \mathrm{CFU} / \mathrm{ml}\right)$ isolated from human dandruff flakes was incubated in rich media in the absence and presence of $20 \mathrm{~g} / \mathrm{l}$ glycerol, $0.0005 \% \mathrm{mPEG}$ diluted in water, or $0.0005 \%$ mPEG-PCL dissolved in $0.5 \%$ acetone at $37^{\circ} \mathrm{C}$. Controls include rich media (with/without acetone) plus glycerol, mPEG, or mPEG-PCL without microbes. The $0.002 \%(\mathrm{w} / \mathrm{v})$ phenol red (Sigma) in rich media with glycerol, mPEG or mPEG-PCL served as an indicator, changing the color from red-orange to yellow due to fermentation. The color change of phenol red from red-orange to yellow was monitored by the decrease in $\mathrm{OD}$ at $560 \mathrm{mM}\left(\mathrm{OD}_{560}\right)$. 
Citation: Kao MS, Wang Y, Marito S, Huang S, Lin WZ, et al. (2016) The mPEG-PCL Copolymer for Selective Fermentation of Staphylococcus lugdunensis Against Candida parapsilosis in the Human Microbiome. J Microb Biochem Technol 8: 259-265. doi: 10.4172/19485948.1000295

\section{Gas chromatography-mass spectrometry (GC/MS)}

S. lugdunensis $\left(10^{5} \mathrm{CFU} / \mathrm{ml}\right)$ was incubated in rich media in the presence of mPEG-PCL $(0.0005 \%)$ for three days. After removing S. lugdunensis by centrifugation at $5,000 \mathrm{~g}$ for $10 \mathrm{~min}$, SCFAs in the fermentation media $(0.5 \mathrm{ml})$ were determined by ethyl acetate (Residue Analysis OmniSolv, EMD Millipore, Billerica, MA) liquid-liquid extraction after addition of the internal standard $\left(0.1 \mathrm{mg} / \mathrm{ml}\right.$ of ${ }^{2} \mathrm{H}_{7}-$ butyric acid, C/D/N Isotopes, Quebec, Canada), acidification with $0.5 \%$ ortho-phosphoric acid (Thermo Fisher Scientific, Fair Lawn, NJ) and saturation with sodium chloride (Thermo Fisher Scientific) followed by GC-MS analysis using an Agilent 5890 Series II GC coupled with 5971 MS detector (Agilent Technologies, Inc., Palo Alto, CA) [28]. A $70 \mathrm{eV}$ electron was used for ionization. Acetic, propionic, isobutyric, butyric, isovaleric and valeric acids were quantified by a calibration curve made from six non-zero levels using the Free Fatty Acids Test Standard (Restek Corporation, Bellefonte, PA).

\section{Fungicidal effects of acetic acid and diethyleneglycol diacetate (Ac-DEG-Ac)}

To determine the fungicidal activities of acetic acid and Ac-DEGAc, a pro-drug with two Ac esterified to a diethylene glycol (DEG) liner, C. parapsilosis $\left(10^{8} \mathrm{CFU}\right.$ in $\left.1 \mathrm{ml} \mathrm{H}_{2} \mathrm{O}\right)$ was incubated overnight with acetic acid in $\mathrm{H}_{2} \mathrm{O}(1 \mathrm{ml})$ or Ac-DEG-Ac in $4 \%$ dimethyl sulfoxide (DMSO) $(1 \mathrm{ml})$ at various concentrations $(0.01-500 \mathrm{mM})$ as indicated in each individual experiment in media in an eppendorf. The controls were kept in $1 \mathrm{ml} \mathrm{H}_{2} \mathrm{O}$ or $4 \%$ DMSO. After incubation, fungi were diluted 1:10-1:105 with $\mathrm{H}_{2} \mathrm{O}$. The percent growth inhibition of $C$. parapsilosis by acetic acid or Ac-DEG-Ac relative to fungi treated with control was determined.

\section{Ex vivo efficacy of acetic acid and Ac-DEG-Ac against fungi}

The human dandruff flakes $\left(>1 \mathrm{~mm}^{2}\right)$ were cut in two halves, and the first half was incubated with acetic acid $\left(10 \mathrm{mM}\right.$ in $\left.\mathrm{H}_{2} \mathrm{O}\right)$ or AcDEG-Ac (10 mM in $4 \%$ DMSO) for $3 \mathrm{~h}$ at room temperature. The other half was incubated with $\mathrm{H}_{2} \mathrm{O}$ or $4 \%$ DMSO as a control. Dandruff flakes were placed on MEA plates and incubated for 4 days at $30^{\circ} \mathrm{C}$. The (a)

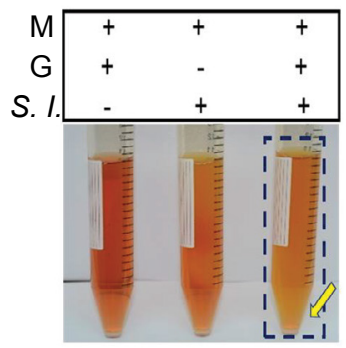

(b)

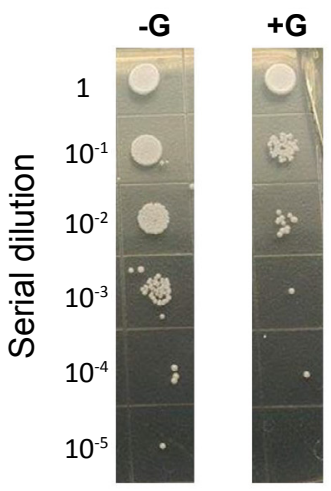

Figure 1: Probiotic activity of $S$. lugdunensis fermentation against $C$. parapsilosis. (a) S. lugdunensis (S. I.) $\left(10^{5} \mathrm{CFU} / \mathrm{ml}\right)$, was incubated in rich media (M) with or without glycerol $(G)$. Rich media plus glycerol without $S$. lugdunensis was included as a control. S. lugdunensis fermentation on 36 $\mathrm{h}$ was displayed. A color change to yellow in the media (marked in a blue frame and yellow arrow) indicates that bacterial fermentation has occurred. (b) S. lugdunensis $\left(10^{5} \mathrm{CFU}\right)$ was co-cultured with $\mathrm{C}$. parapsilosis $\left(10^{5} \mathrm{CFU}\right)$ in rich media $(10 \mathrm{ml})$ in the presence $(+G)$ or absence $(-G)$ of glycerol $(20$ $\mathrm{g} / \mathrm{l})$. After 3 day culture, media $(10 \mu \mathrm{l})$ with a serial dilution $\left(1-10^{5}\right)$ were spotted on furazolidone $(10 \mu \mathrm{g} / \mathrm{ml})$ containing PDA plates for three days. Data representative of three separate experiments are shown. change in the area $\left(\mathrm{mm}^{2}\right)$ of fungal growth in dandruffs was measured and calculated with ImageJ software (NIH, Bethesda, MD, USA). More than three dandruff flakes per group experiment were used.

\section{Statistical analysis}

To determine significances between groups, comparisons were made using the two-tailed $t$-test. For all statistical tests, the $P$-values of $<0.05\left(^{*}\right),<0.01\left({ }^{* *}\right)$, and $<0.001\left(^{* * *}\right)$ were accepted for statistical significance.

\section{Results}

\section{Inhibition of $C$. parapsilosis growth by glycerol fermentation of $S$. lugdunensis}

Four bacteria (S. lugdunensis, S. epidermidis, Staphylococcus warneri and Staphylococcus capitis) and two fungi (C. parapsilosis and Penicillium citrinum) were identified and isolated from human dandruff flakes (Supplementary Table 1). To examine the fermentation activities of these bacteria and fungi, each individual microbe was incubated in rich media in the presence of $20 \mathrm{~g} / \mathrm{l}$ glycerol, a naturally occurring metabolite found in human skin [29], as the carbon source. Rich media plus glycerol and rich media plus microbes were used as controls. To observe the fermentation process, rich media were added with phenol red, a fermentation indicator, to assess SCFA production as a result of glycerol fermentation. Consistent with our previous study [16], media in the culture of $S$. epidermidis with glycerol turned yellow which is the result of acid production three days following incubation (data not shown), demonstrating microbial fermentation. Besides S. epidermidis, the S. lugdunensis (Figure 1a) and C. parapsilosis (Supplementary Figure 1) were two microbes in human dandruff that can elicit fermentation of glycerol, making media turn yellow 36 and $96 \mathrm{~h}$ after incubation, respectively. S. lugdunesis is a normal inhabitant of the human skin. Previous studies demonstrated the isolation of $C$. parapsilosis from the human scalps and grew this fungus out along hair shafts planted in primary isolation media [30]. Our results here endorse that $S$. lugdunesis and C. parapsilosis co-exist in the human dandruff.

To investigate if $S$. lugdunesis fermentation influences the growth of C. parapsilosis, S. lugdunesis was co-cultured with C. parapsilosis in the presence or absence of glycerol for three days. To establish a C. parapsilosis-selective plate, media $(10 \mu \mathrm{l})$ from the co-culture of $S$. lugdunesis and C. parapsilosis was spotted on a PDA plate supplemented with $10 \mu \mathrm{g} / \mathrm{ml}$ furazolidone. We found that $10 \mu \mathrm{g} / \mathrm{ml}$ furazolidone can completely kill $S$. lugdunesis without affecting the growth of $C$. parapsilosis (Supplementary Figure 2). Three days after microbial co-culture with/without glycerol, media with serial dilutions $\left(1-10^{5}\right)$ were spotted on $C$. parapsilosis-selective plates. The numbers of $C$. parapsilosis in the co-culture in the absence of glycerol found were at least one log order of magnitude greater than those in the co-culture in the presence of glycerol (Figure 1b). These data in Figure 1 suggest that S. lugdunesis mediates the glycerol fermentation to hamper the growth of C. parapsilosis.

\section{Synthesis of $\mathrm{mPEG}$-PCL as a selective fermentation initiator of S. lugdunensis}

The polymerization was catalyzed by the addition of stannous 2-ethylhexanoate. Upon the complexation of monomer $\varepsilon$-caprolactone with the catalyst, the nucleophilic mPEG reacted with the monomer $\varepsilon$-caprolactone to give the product mPEG-PCL [31]. The purified polymer of $\mathrm{mPEG}-\mathrm{PCL}$ was a white powder. The number-average $\mathrm{Mw}$ and weight-average Mw of PEG-PCL were 5,182 and 9,767 Da, 
Citation: Kao MS, Wang Y, Marito S, Huang S, Lin WZ, et al. (2016) The mPEG-PCL Copolymer for Selective Fermentation of Staphylococcus lugdunensis Against Candida parapsilosis in the Human Microbiome. J Microb Biochem Technol 8: 259-265. doi: 10.4172/19485948.1000295

respectively, leading to a polydispersity of 1.885 . The functional groups revealed in a FT-IR spectrum were found at wave numbers of $1,727.9$ $\mathrm{cm}^{-1}$ for $\mathrm{C}=\mathrm{O}$ on PCL block and $1,184.1 \mathrm{~cm}^{-1}$ for $\mathrm{C}-\mathrm{O}-\mathrm{C}$ on $\mathrm{mPEG}$ block, respectively (Figure 2a). The melting point determined by DSC was at $53.6^{\circ} \mathrm{C}$. Compared to our previous paper where the melting point of PEG-PCL having number-average Mw of $17,217 \mathrm{Da}$ was $57.2^{\circ} \mathrm{C}$, the mPEG-PCL used in the current study has lower $\mathrm{Mw}$ and yet lower melting point [32].

PEG-derived polymers have been employed as a carbon source for microbial fermentation [23]. Different microbial species make different enzymes that ferment specific substrates. To examine whether S. lugdunesis and C. parapsilosis differentially utilizes the mPEG or mPEG-PCL for fermentation, S. lugdunesis or C. parapsilosis was incubated in rich media in the absence or presence of $0.0005 \% \mathrm{mPEG}$ PCL. Controls include rich media with mPEG alone, mPEG-PCL alone or microbes alone. Incubation of S. lugdunesis with $0.0005 \% \mathrm{mPEG}$ for $36 \mathrm{~h}$ did not induce fermentation (Supplementary Figure 3). As shown in Figure 2b, mPEG-PCL selectively triggered S. lugdunesis, but not $C$. parapsilosis, to undergo fermentation. In the culture of S. lugdunesis with $\mathrm{mPEG}$-PCL, the phenol red-containing media started turning yellow $36 \mathrm{~h}$ after culture. The $\mathrm{OD}_{560} / \mathrm{pH}$ values of media with mPEGPCL alone, S. lugdunesis alone or mPEG-PCL plus $S$. lugdunesis for $36 \mathrm{~h}$ are $0.53 \pm 0.02 / 7.36 \pm 0.01 ; 0.42 \pm 0.01 / 7.12 \pm 0.02$; and $0.32 \pm 0.01 / 6.88$ \pm 0.01 . A significant decrease in $\mathrm{OD}_{560}$ and $\mathrm{pH}$ values in the media with mPEG-PCL plus $S$. lugdunesis indicated the mPEG-PCL fermentation of $S$. lugdunesis. No yellow media in the culture of C. parapsilosis with mPEG-PCL were detected. The mPEG-PCL was thus chosen as a selective fermentation initiator of $S$. lugdunensis. The exogenous addition of mPEG-PCL may be able to enhance the probiotic activities of $S$. lugdunensis for suppression of the growth of C. parapsilosis in human dandruff.

\section{Identification of SCFAs produced by mPEG-PCL fermentation by GC/MS}

To identify the SCFAs in the ferments, the S. lugdunensis were incubated in phenol red-free rich media in the presence of mPEGPCL $(0.0005 \%)$ for two days. Supernatants of bacterial culture $S$. lugdunensis mixed with ${ }^{2} \mathrm{H}_{7}$-butyric acid (an internal standard)

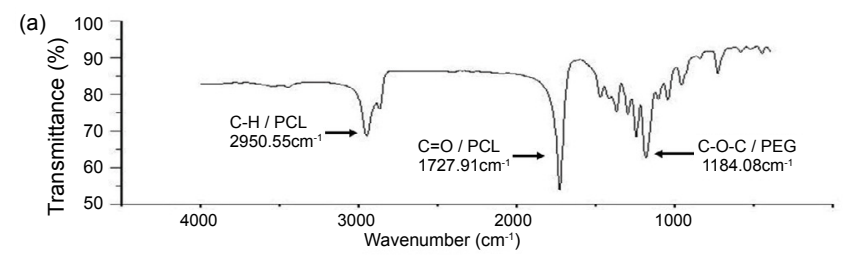

(b)

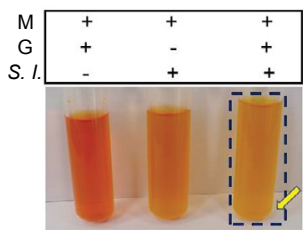

(c)

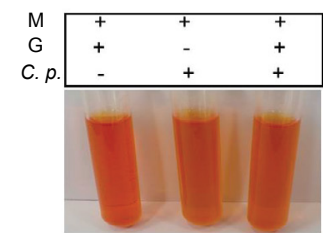

Figure 2: Characterizations of mPEG-PCL as a selective fermentation initiator of S. lugdunensis fermentation. (a) Three functional groups (C-O-C/ $\mathrm{PEG} / 1184.1 \mathrm{~cm}^{-1} ; \mathrm{C}=\mathrm{O} / \mathrm{PCL} / 1727.9 \mathrm{~cm}^{-1}$; and C-H/PCL/2950.6 $\mathrm{cm}^{-1}$ ) of $\mathrm{mPEG}-$ PCL were revealed in a FT-IR spectrum. (b) $S$. lugdunensis, but not (c) $C$ parapsilosis, fermented mPEG-PCL. $0.0005 \% \mathrm{mPEG}-\mathrm{PCL}$ in $0.5 \%$ acetone was used as a carbon source for fermentation. A blue frame and yellow arrow denote the mPEG-PCL fermentation of the S. lugdunensis. Representative data from three independent experiments are shown. were subjected to GC-MS analysis. Two major SCFAs (acetic acid and isovaleric acid) in the fermented media of S. lugdunensis and ${ }^{2} \mathrm{H}_{7}$-butyric acid were detected by GC separation (Figure 3a). Mass spectra of acetic acid (Figure $3 \mathrm{~b}$ ) and isovaleric acid (data not shown) were subsequently generated. Molecular ions at 29, 43, 45 and $60 \mathrm{~m} / \mathrm{z}$ values corresponding to $[\mathrm{HCO}]^{+},\left[\mathrm{CH}_{3} \mathrm{CO}\right]^{+},\left[\mathrm{CH}_{3} \mathrm{CO}\right]^{+}, \mathrm{CH}_{3} \mathrm{COOH}$ for acetic acid are detected in a MS spectrum (Figure $3 \mathrm{~b}$ ). These results demonstrate that $S$. lugdunensis fermentatively metabolized mPEG-PCL into SCFAs.

\section{Suppression of the $C$. parapsilosis growth by acetic acid and Ac-DEG-Ac}

Two pharmacokinetic drawbacks of SCFAs as drugs are associated with their rapid metabolization and inability to accomplish effective concentrations in vivo $[33,34]$. Thus, Ac-DEG-Ac, a pro-drug of acetic acid (Ac) which contains two acetic acids esterified to a DEG linker, was included for evaluation of its fungicidal activity against $C$. parapsilosis. To compare Ac-DEG-Ac to acetic acid, C. parapsilosis was incubated with the concentrations (0.01-500 mM) of acetic acid (Figures $4 \mathrm{a}$ and $4 \mathrm{~b}$ ) in $\mathrm{H}_{2} \mathrm{O}$ or Ac-DEG-Ac (Figures $4 \mathrm{c}$ and $4 \mathrm{~d}$ ) in DMSO overnight. C. parapsilosis incubated with $\mathrm{H}_{2} \mathrm{O}$ or DMSO served as controls. Both acetic acid and Ac-DEG-Ac at concentrations in the range of 0.01$10 \mathrm{mM}$ inhibited approximately $50 \%$ growth of $C$. parapsilosis. Both agents at a concentration of $100 \mathrm{mM}$ suppressed the growth of $C$. parapsilosis by greater than $90 \%$ and completely killed C. parapsilosis at a concentration of $500 \mathrm{mM}$, suggesting that both acetic acid and AcDEG-Ac exert anti-C. parapsilosis activities in vitro.

\section{Fungicidal activities of acetic acid and Ac-DEG-Ac against fungi in human dandruff}

Filamentous microbes containing different fungi are typically found in human dandruffs. Besides anti-C. parapsilosis properties, we next examined if acetic acid and Ac-DEG-Ac can inhibit the growth of various fungi in human dandruffs. A human dandruff flake was cut in two halves, and the first half was incubated with $10 \mathrm{mM}$ acetic acid or $10 \mathrm{mM}$ Ac-DEG-Ac. The other half was incubated with $\mathrm{H}_{2} \mathrm{O}$ or DMSO as controls. Four days after incubation, we found that both acetic acid and Ac-DEG-Ac can efficiently block the extension of fungal growth in human dandruffs. The result suggests that acetic acid and Ac-DEGAc may have a broad spectrum of anti-fungal activity for treatment of human dandruff.

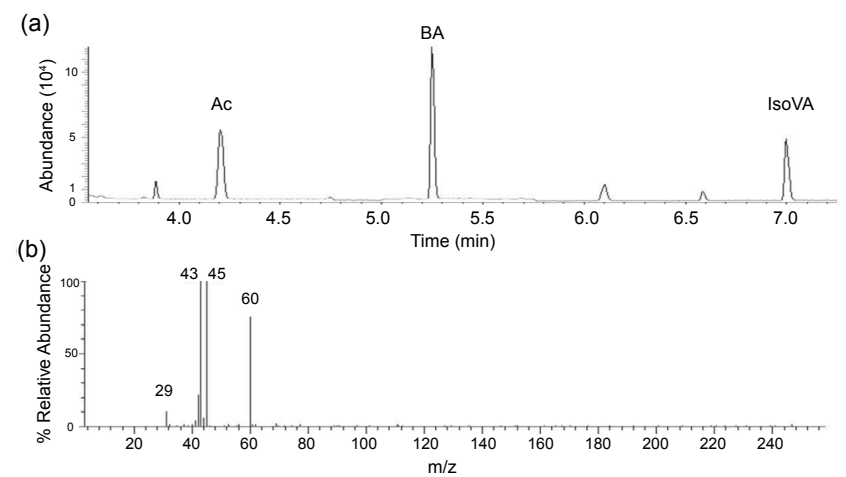

Figure 3: The ion chromatogram and mass spectrum from GC-MS for identification of SCFAs. (a) Total ion chromatogram for separation of the mixture of SCFAs containing acetic acid (Ac), ${ }^{2} \mathrm{H}_{7}$-butyric acid (BA) (an internal standard) and isovaleric acid (IsoVa). (b) A mass spectrum for acetic acid. Molecular ions at 29, 43, 45 and $60 \mathrm{~m} / \mathrm{z}$ for acetic acid were indicated. 
Citation: Kao MS, Wang Y, Marito S, Huang S, Lin WZ, et al. (2016) The mPEG-PCL Copolymer for Selective Fermentation of Staphylococcus lugdunensis Against Candida parapsilosis in the Human Microbiome. J Microb Biochem Technol 8: 259-265. doi: 10.4172/19485948.1000295

\section{Discussion}

At least three new findings are presented in the current study. First, S. lugdunensis was found to be a probiotic bacterium which can ferment glycerol and mPEG-PCL. Second, the mPEG-PCL was used for the first time as a selective fermentation initiator to exclusively trigger the fermentation of $S$. lugdunensis against $C$. parapsilosis. The mPEG-PCL, PEG- or PCL-derived polymers can be developed as drug adjuvants in the future to reduce the amount of active drugs while maintaining the antimicrobial activity, decreasing the risk of generating resistant microbes. Third, the fungicidal activity of AcDEG-Ac demonstrates a successful approach for development of novel drugs from the resources of fermentation metabolites of skin probiotic bacteria. Yogurts containing live probiotic strains, the best examples of bacterial interference, have been used for centuries to maintain the digestive microbial ecosystem. Skin probiotic bacteria characterized in our previous papers can operate fermentation to suppress the pathogen colonization [15]. S. lugdunensis, a coagulase-negative staphylococcus, is a common colonizer of the human skin, and it is the only pathogen in about $10 \%$ of skin and soft tissue infections [35,36]. C. parapsilosis, a human opportunistic fungus, is frequently isolated from human skin [37]. It has been shown that C. parapsilosis can be isolated from human scalp [30]. The fungus has been recognized as commensal yeasts on the dog skin but also a causative microbe of seborrheic dermatitis, particularly in atopic dogs [38]. Although the clinical evidence about the oppositional relationship between S. lugdunensis and C. parapsilosis in the human skin is lacking, our data revealed that $S$. lugdunensis can exploit glycerol fermentation to hinder the growth of C. parapsilosis in vitro (Figure 1). During the development of dandruff, S. lugdunensis and $C$. parapsilosis may use the glycerol as a shared carbon source and produce different SCFAs as (antimicrobial) weapons to repel each other on the human scalp. C. parapsilosis may "win the battle" of microbial interference when the dandruff is persistent. Our strategy to cure dandruff is to deter the growth of C. parapsilosis by amplification of the probiotic activities of $S$. lugdunensis using a selective fermentation initiator.

PEG is a synthetic water-soluble polymer of the common structural formula $\mathrm{H}\left(\mathrm{OCH}_{2} \mathrm{CH}_{2}\right)_{\mathrm{n}} \mathrm{OH}$. An extracellular enzyme which can (a)

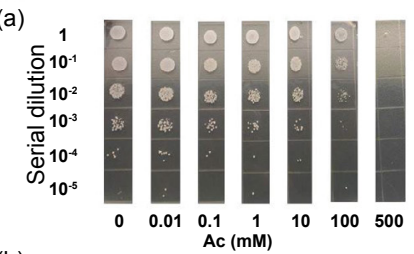

(b)

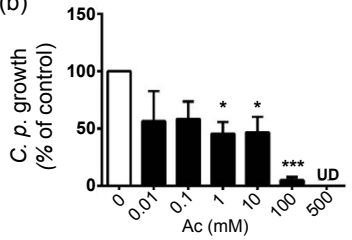

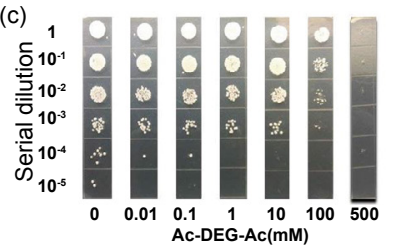

(d)

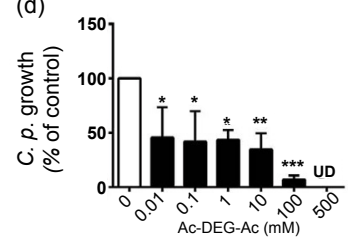

Figure 4: Suppression of $C$. parapsilosis growth by acetic acid and Ac-DEGAc. C. parapsilosis $\left(10^{8} \mathrm{CFU}\right)$ was incubated with $0.01-500 \mathrm{mM}$ acetic acid in $\mathrm{H}_{2} \mathrm{O}(\mathrm{a}, \mathrm{b})$ or Ac-DEG-Ac in $4 \%$ DMSO (c, d) overnight. Incubation of $C$ parapsilosis with $\mathrm{H}_{2} \mathrm{O}$ or $4 \%$ DMSO served as controls. After incubation, $C$. parapsilosis was diluted $1: 10-1: 10^{5}$ with $\mathrm{H}_{2} \mathrm{O}$, and $10 \mu \mathrm{l}$ of the dilutions were spotted on an agar plate. Percent growth inhibition of $C$. parapsilosis relative to the treatment with $\mathrm{H}_{2} \mathrm{O}$ control was presented. The CFU counts were illustrated as the mean \pm standard derivation (SD) of six independent experiments $(b, d)$. ${ }^{\star * *} P<0.001$; ${ }^{* \star} P<0.01 ;{ }^{*} P<0.05$, (two-tailed t-tests). UD, undetectable. depolymerize long chain PEGs was identified [39]. The diol dehydratase and PEG acetaldehyde lyase in fermenting bacteria can degrade PEG [40]. However, results from other laboratories demonstrate that PEG degradation by fermenting bacteria was inhibited by ethylene glycol, probably owing to a blocking of the cellular uptake system. The results suggest that PEG was taken up into the bacteria and subsequently degraded inside [23]. The depolymerization of PEG can be catalyzed by bacteria via hydrolysis after a modification of the terminal ethylene glycol (EG) unit [23]. PEG fermentations yield acetaldehyde as an intermediate, which is further disproportionated to acetate and ethanol. A propionate-forming bacterium can ferment PEG to acetate and propionate [41]. PCL was found to be degraded by lipase from various sources and cutinase from a fungus $[42,43]$. PCL-degrading bacteria are widely distributed in nature. PCL depolymerase detected in both extracellular and intracellular fractions of bacterial cultures can efficiently degrade PCL [44]. No one reported that bacteria can use mPEG-PCL as a carbon source for fermentation. Our results demonstrate for the first time that $S$. lugdunensis, but not C. parapsilosis, can fermentatively metabolize mPEG-PCL to acetate and isovaleric acid (Figure 3). Since both S. lugdunensis and C. parapsilosis may use the same carbon source [e.g. glycerol; (Figure 1 and Supplementary Figure 1)] outcompete each other to survive. Although it is unclear why $S$. lugdunensis can fermeatatively metabolize mPEG-PCL, but not mPEG (Supplementary Figure 3), it is possible that mPEG-PCL containing more carbon atoms than mPEG provides a relatively abundant carbon source for $S$. lugdunensis fermentation. In addition, although incubation of $C$. parapsilosis with mPEG-PCL for $96 \mathrm{~h}$ did not induce fermentation (Figure 2c), it is worth investigate whether C. parapsilosis can non-fermentatively degrade mPEG-PCL to metabolites that affect the growth of $S$. lugdunensis. Our results support that the mPEG-PCL can function as a selective fermentation initiator to specially amplify the fermentation activity of $S$. lugdunensis against $C$. parapsilosis. Therefore, mPEG-PCL holds promise as a manipulation technique to reduce the fungal over-growth in the dysbiotic dandruff.

Phage therapy is yet another manipulation technique, by which selective overgrown microbes can be targeted with specific bacteriophages, thereby normalizing the dysbiotic skin microbiome. Although specific bacteriophages for C. parapsilosis have been not yet identified, the disadvantage of phage therapy includes the potential ability of bacteriophages to transfer the DNA from a microbe to another. This DNA transfer could be in charge of the transfer of pathogenicity determinants and virulence factors, resulting in the generation of even more resistant microbial strains [45-47]. Although the toxicity of mPEG-PCL to the skin has not been evaluated, the mPEG-PCL, a highly biocompatible and biodegradable copolymer, has been widely used as a component of nanoparticles for transdermal delivery [48]. Most importantly, both PEG and PCL have been approved by the FDA in specific applications used in humans as a drug delivery device. Thus, the development of mPEG-PLC as a targeted intervention may be relatively safe when it is employed to re-establish healthy patterns of bacterial-fungal interactions in the dysbiotic skin microbiome. Potentially, the mPEG-PCL can function as a drug adjuvant to augment the fermentation activity of skin probiotic bacteria and reduce the effective doses of fungicides in the future. The use of mPEG-PCL analog with lower dose of fungicides may minimize the risk of development of resistant fungi and the non-specific killing effect of fungicides on skin commensals.

The SCFAs produced by fermentation of intestinal microbes in the human colon can reach a high level in the $20-140 \mathrm{mM}$ range that can effectively ward off local pathogens [49]. However, the amounts of 
Citation: Kao MS, Wang Y, Marito S, Huang S, Lin WZ, et al. (2016) The mPEG-PCL Copolymer for Selective Fermentation of Staphylococcus lugdunensis Against Candida parapsilosis in the Human Microbiome. J Microb Biochem Technol 8: 259-265. doi: 10.4172/19485948.1000295

SCFAs in peripheral circulation are relatively low, ranging from 3 to 7 $\mu \mathrm{M}[49]$. Numerous SCFAs are frequently detected in the skin and in the secretions of skin glands, such as the sweat, but their concentrations are generally low. For example, sweat only contains $0.0096 \%$ acetic acid [50]. Although the levels of SCFAs in human scalp skin have yet to be quantified, it has been documented that SCFAs have short half-lives and the apparent difficulty of achieving pharmacologic concentrations when they are administered in vivo. Furthermore, although SCFA is a normal human metabolite and theoretically less toxic, SCFA at high doses may create an extremely acid solution, which may be toxic to skin cells. A butyric acid pro-drug named pivaloylomethyl butyrate (AN-9) has been developed to achieve an effective concentration of butyric acid [51]. To increase the half-life of propionic acid, we have synthesized a pro-drug of propionic acid by esterifying two active propionic acids to a DEG linker. The pro-drug of propionic acid exerts excellent antimicrobial activity against $S$. aureus [49]. In this study, we demonstrate that a pro-drug of acetic acid (Ac-DEG-Ac) that is relatively less acidic and contains two acetic acid moieties esterified to a DEG linker efficiently suppresses the growth of fungi in the human dandruff (Figure 5).

Esterases such as carboxylic esterase are capable of hydrolyzing the ester group of esterified pro-drug [52]. Although esterases are expressed by host cells, esterases in extracellular fluids or released from microbes have been identified [53-56]. Dead cells such as dandruff may also release esterases [57,58]. Future studies will determine if the Ac-DEGAc is cleaved by esterases released from microbes and/or dead cells to increase the local concentrations of acetic acids, thereby becoming pharmacologically effective to decolonize the fungi in dandruff flakes. We also cannot rule out the possibility of anti-fungal activity of uncleaved Ac-DEG-Ac. Although it remains unclear how SCFAs impede the fungal growth, prior findings suggested that a lowered intracellular $\mathrm{pH}$ value of microbes is a lethal mechanism of SCFA [59]. Mounting evidence has demonstrated the anti-inflammatory effect of SCFAs [60]. The anti-inflammatory effect of SCFAs may serve to promote immunological tolerance to commensal bacteria via stimulation of immunosuppressive interleukin (IL) 10 production of regulatory $\mathrm{T}\left(\mathrm{T}_{\text {reg }}\right)$ cells, maximizing the probiotic activities of skin commensal bacteria to outcompete the over-growth of pathogens. (a)

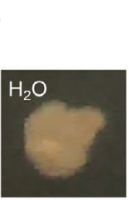

(b)
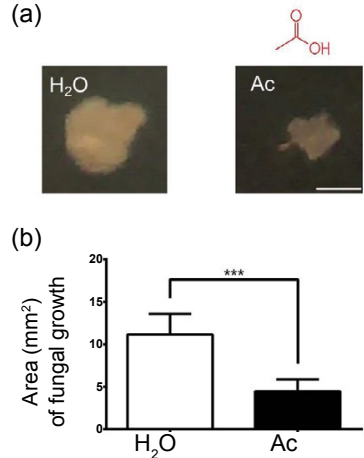

(c)

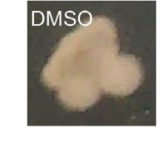

(d)

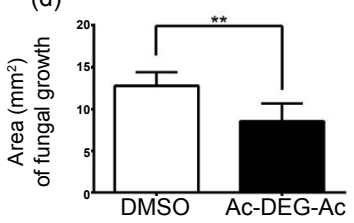

Figure 5: Inhibition of fungal growth in human dandruffs by acetic acid and Ac-DEG-Ac. A human dandruff flake was cut in half, and half was incubated with acetic acid in $\mathrm{H}_{2} \mathrm{O}(\mathrm{Ac})(\mathrm{a}, \mathrm{b})$ or Ac-DEG-Ac (c, d) in DMSO for $3 \mathrm{~h}$. The other half was incubated with $\mathrm{H}_{2} \mathrm{O}$ or DMSO as a control. Both chemical structures of acetic acid and Ac-DEG-Ac were illustrated. (b) The sizes $\left(\mathrm{mm}^{2}\right)$ of fungal growth in dandruffs treated with acetic acid, Ac-DEG-Ac or their controls were quantified 4 days after placing dandruffs on MEA plates. At least three dandruff flakes per group experiment were used. ${ }^{\star * *} P<0.001 ;{ }^{* *} P<0.01$, (two-tailed t-tests). Bars $=2.0 \mathrm{~mm}$.
Thus, it is worth exploring the impacts of acetic acid and Ac-DEG-Ac on the host inflammatory responses and long-term homeostasis of the human skin microbiome.

Lack of selectivity and induction of resistance are central problems for many fungicides. Vaccines, although the actions are fairly specific, may become less potent for treatments of patients with significant underlying health matters such as diabetes, surgical intervention, and immune suppression. In contrast, probiotic treatments, which are relatively independent on patients' health matters, have little or no interference with commensal microbes may complement fungicides or vaccines for treatments of fungal infections in the skin.

\section{Conclusion}

Here, we envision that the interference of bacteria with the growth of fungi via fermentation naturally occurs in the human scalp skin. The use of endogenous molecules, such as SCFAs in fermentation products, as natural fungicides is concordant with evolutionary medicine and provides a new set of tools for fighting the fungal resistance.

\section{Acknowledgement}

This work was supported by a National Institutes of Health (NIH) STTR grant (1R41AR065260-01A1) and a Ministry of Science and Technology (MOST) grant (104-2221-E-033-068). We appreciate Dr. Roland Kirschner's laboratory for assistance with the D1/D2 5.8S rRNA gene sequencing. We also would like to thank Sunita Keshari for her great effort for editing this manuscript.

\section{References}

1. SanMiguel A, Grice EA (2015) Interactions between host factors and the skin microbiome. Cell Mol Life Sci 72: 1499-1515.

2. Wang L, Clavaud C, Bar-Hen A, Cui M, Gao J, et al. (2015) Characterization of the major bacterial-fungal populations colonizing dandruff scalps in Shanghai, China, shows microbial disequilibrium. Exp Dermatol 24: 398-400.

3. Park HK, Ha MH, Park SG, Kim MN, Kim BJ, et al. (2012) Characterization of the fungal microbiota (mycobiome) in healthy and dandruff-afflicted human scalps. PLoS One 7: e32847.

4. Kobayashi T, Glatz M, Horiuchi K, Kawasaki H, Akiyama H, et al. (2015) Dysbiosis and Staphylococcus aureus Colonization Drives Inflammation in Atopic Dermatitis. Immunity 42: 756-766.

5. Wang L, Clavaud C, Bar-Hen A, Cui M, Gao J, et al. (2015) Characterization of the major bacterial-fungal populations colonizing dandruff scalps in Shanghai, China, shows microbial disequilibrium. Exp Dermatol 24: 398-400.

6. Nowicki R (2006) [Modern management of dandruff]. Pol Merkur Lekarski 20: 121-124.

7. Turner GA, Hoptroff M, Harding CR (2012) Stratum corneum dysfunction in dandruff. Int J Cosmet Sci 34: 298-306.

8. Wei W, Cao Z, Zhu YL, Wang X, Ding G, et al. (2006) Conserved genes in a path from commensalism to pathogenicity: comparative phylogenetic profiles of Staphylococcus epidermidis RP62A and ATCC12228. BMC Genomics 7: 112.

9. Ji G, Beavis R, Novick RP (1997) Bacterial interference caused by autoinducing peptide variants. Science 276: 2027-2030.

10. Otto M (2009) Staphylococcus epidermidis--the 'accidental' pathogen. Nat Rev Microbiol 7: 555-567.

11. Whitehead SS, Leavitt RW, Jensen MM (1993) Staphylococcosis of turkeys. 6 Development of penicillin resistance in an interfering strain of Staphylococcus epidermidis. Avian Dis 37: 536-541.

12. Nicoll TR, Jensen MM (1987) Avian diseases 31: 85-88.

13. Wilkinson DM, Jensen MM (1987) Staphylococcosis of turkeys. 4 Characterization of a bacteriocin produced by an interfering Staphylococcus. Avian Dis 31: 80-84.

14. Shinefield HR, Ribble JC, Boris M (1971) Bacterial interference between strains of Staphylococcus aureus, 1960 to 1970. Am J Dis Child 121: 148-152.

15. Shu M, Wang Y, Yu J, Kuo S, Coda A, et al. (2013) Fermentation of 
Citation: Kao MS, Wang Y, Marito S, Huang S, Lin WZ, et al. (2016) The mPEG-PCL Copolymer for Selective Fermentation of Staphylococcus lugdunensis Against Candida parapsilosis in the Human Microbiome. J Microb Biochem Technol 8: 259-265. doi: 10.4172/19485948.1000295

Propionibacterium acnes, a commensal bacterium in the human skin microbiome, as skin probiotics against methicillin-resistant Staphylococcus aureus. PLoS One 8: e55380.

16. Wang Y, Kuo S, Shu M, Yu J, Huang S, et al. (2014) Staphylococcus epidermidis in the human skin microbiome mediates fermentation to inhibit the growth of Propionibacterium acnes: implications of probiotics in acne vulgaris. Appl Microbiol Biotechnol 98: 411-424

17. Ushijima T, Takahashi M, Ozaki Y (1984) Acetic, propionic, and oleic acid as the possible factors influencing the predominant residence of some species of Propionibacterium and coagulase-negative Staphylococcus on normal human skin. Can J Microbiol 30: 647-652.

18. Ryssel H, Kloeters O, Germann G, Schäfer T, Wiedemann G, et al. (2009) The antimicrobial effect of acetic acid--an alternative to common local antiseptics? Burns 35: 695-700.

19. Sebastian S, Phillip LE, Fellner V, Idziak ES (1996) Comparative assessment of bacterial inoculation and propionic acid treatment of aerobic stability and microbial populations of ensiled high-moisture ear corn. J Anim Sci 74: 447-456.

20. Retraction. Transplantation: tolerance (Current Opinion in Investigational Drugs (2003) 4: 530-535). Curr Opin Investig Drugs 10: 882

21. Gupta AK, Nicol K (2004) The use of sulfur in dermatology. J Drugs Dermato 3: $427-431$.

22. Liss RH, Batchelor FR (1987) Economic evaluations of antibiotic use and resistance--a perspective: report of Task Force 6. Rev Infect Dis 9 Suppl 3: S297-312.

23. Schink B, Stieb M (1983) Fermentative degradation of polyethylene glycol by a strictly anaerobic, gram-negative, non-sporeforming bacterium, Pelobacter venetianus sp. nov. Appl Environ Microbiol 45: 1905-1913.

24. Manuel F, Ranganathan S (2011) A new postulate on two stages of dandruff: a clinical perspective. Int J Trichology 3: 3-6.

25. Singh R, Parija SC (2012) Candida parapsilosis: an emerging fungal pathogen. Indian J Med Res 136: 671-673.

26. Lindh JM, Terenius O, Faye I (2005) 16S rRNA gene-based identification of midgut bacteria from field-caught Anopheles gambiae sensu lato and $A$. funestus mosquitoes reveals new species related to known insect symbionts. Appl Environ Microbiol 71: 7217-7223.

27. Kurtzman CP, Robnett CJ (1997) Identification of clinically importan ascomycetous yeasts based on nucleotide divergence in the 5 ' end of the largesubunit (26S) ribosomal DNA gene. J Clin Microbiol 35: 1216-1223.

28. García-Villalba R, Giménez-Bastida JA, García-Conesa MT, Tomás-Barberán FA, Carlos Espín J, et al. (2012) Alternative method for gas chromatographymass spectrometry analysis of short-chain fatty acids in faecal samples. J Sep Sci 35: 1906-1913.

29. Fluhr JW, Darlenski R, Surber C (2008) Glycerol and the skin: holistic approach to its origin and functions. Br J Dermatol 159: 23-34.

30. Taj-Aldeen SJ, Al-Ansari HI, Boekhout T, Theelen B (2004) Co-isolation of Trichosporon inkin and Candida parapsilosis from a scalp white piedra case. Med Mycol 42: 87-92

31. Labet M, Thielemans W (2009) Synthesis of polycaprolactone: a review. Chem Soc Rev 38: 3484-3504.

32. Jiang CP, Chen YY, Hsieh MF3 (2013) Biofabrication and in vitro study of hydroxyapatite/mPEG-PCL-mPEG scaffolds for bone tissue engineering using air pressure-aided deposition technology. Mater Sci Eng C Mater Biol Appl 33: 680-690.

33. Schröder O, Opritz J, Stein J (2000) Substrate and inhibitor specificity of butyrate uptake in apical membrane vesicles of the rat distal colon. Digestion 62: 152-158.

34. Stein J, Zores M, Schröder O (2000) Short-chain fatty acid (SCFA) uptake into Caco-2 cells by a pH-dependent and carrier mediated transport mechanism. Eur J Nutr 39: 121-125.

35. Bieber L, Kahlmeter G (2010) Staphylococcus lugdunensis in several niches of the normal skin flora. Clin Microbiol Infect 16: 385-388.

36. Sjolund M, Kahlmeter G (2008) Staphylococci in primary skin and soft tissue infections in a Swedish county. Scand J Infect Dis 40: 894-898.

37. De Bernardis F, Mondello F, San Millàn R, Pontòn J, Cassone A (1999)
Biotyping and virulence properties of skin isolates of Candida parapsilosis. $J$ Clin Microbiol 37: 3481-3486.

38. Yurayart C, Nuchnoul N, Moolkum P, Jirasuksiri S, Niyomtham W, et al (2013) Antifungal agent susceptibilities and interpretation of Malassezia pachydermatis and Candida parapsilosis isolated from dogs with and without seborrheic dermatitis skin. Med Mycol 51: 721-730.

39. Haines JR, Alexander M (1975) environmental microbiology 58: 2164

40. Wagener S, Schink B (1988) Fermentative degradation of non-ionic surfactants and polyethylene glycol by enrichment cultures and by pure cultures of homoacetogenic and propionate-forming bacteria. Appl Environ Microbiol 54: 561-565.

41. Tokiwa Y, Suzuki T (1977) Hydrolysis of polyesters by lipases. Nature 270: 76-78.

42. Murphy CA, Cameron JA, Huang SJ, Vinopal RT (1996) Fusarium polycaprolactone depolymerase is cutinase. Appl Environ Microbiol 62: 456460

43. Oda Y, Oida N, Urakami T, Tonomura K (1997) Polycaprolactone depolymerase produced by the bacterium Alcaligenes faecalis. FEMS Microbiol Lett 152: 339 343.

44. Brabban AD, Hite E, Callaway TR (2005) Evolution of foodborne pathogens via temperate bacteriophage-mediated gene transfer. Foodborne Pathog Dis 2: 287-303.

45. O'Shea YA, Boyd EF (2002) Mobilization of the Vibrio pathogenicity island between Vibrio cholerae isolates mediated by CP-T1 generalized transduction. FEMS Microbiol Lett 214: 153-157.

46. Maiques E, Ubeda C, Tormo MA, Ferrer MD, Lasa I, et al. (2007) Role of staphylococcal phage and SaPI integrase in intra- and interspecies SaPI transfer. J Bacteriol 189: 5608-5616.

47. Che J, Okeke Cl, Hu ZB, Xu J (2015) DSPE-PEG: a distinctive component in drug delivery system. Curr Pharm Des 21: 1598-1605.

48. Garland SH (2011) Short chain fatty acids may elicit an innate immune response from preadipocytes: a potential link between bacterial infection and inflammatory diseases. Med Hypotheses 76: 881-883.

49. Burtenshaw JM (1942) The Journal of hygiene 42: 184-210.

50. Hobdy E, Murren J (2004) AN-9 (Titan). Curr Opin Investig Drugs 5: 628-634.

51. Hirayama F, Ogata T, Yano H, Arima H, Udo K, et al. (2000) Release characteristics of a short-chain fatty acid, n-butyric acid, from its betacyclodextrin ester conjugate in rat biological media. J Pharm Sci 89: 1486-1495.

52. Wang Y, Dai A, Huang S, Kuo S, Shu M, et al. (2014) Propionic acid and its esterified derivative suppress the growth of methicillin-resistant Staphylococcus aureus USA300. Benef Microbes 5: 161-168.

53. Jewell C, Prusakiewicz JJ, Ackermann C, Payne NA, Fate G, et al. (2007) Hydrolysis of a series of parabens by skin microsomes and cytosol from human and minipigs and in whole skin in short-term culture. Toxicol Appl Pharmaco 225: $221-228$.

54. Vincent D, Perrier H, Traeger J (1971) [Carboxylic esterases (cholinesterase and arylesterase) of human lymph of the thoracic duct]. C R Seances Soc Biol Fil 165: 1074-1079.

55. Barr F, Clark H, Hawgood S (1998) Identification of a putative surfactant convertase in rat lung as a secreted serine carboxylesterase. Am J Physio 274: L404-410.

56. Tesch C, Nikoleit K, Gnau V, Götz F, Bormann C (1996) Biochemical and molecular characterization of the extracellular esterase from Streptomyces diastatochromogenes. J Bacteriol 178: 1858-1865.

57. Kato K, Agatsuma T, Tanabe T, Masuko T, Hashimoto Y (1991) Release of esterase from murine lymphokine-activated killer cells in antibody-dependent cellular cytotoxic reaction. Jpn J Cancer Res 82: 206-212.

58. Ostling CE, Lindgren SE (1993) Inhibition of enterobacteria and Listeria growth by lactic, acetic and formic acids. J Appl Bacteriol 75: 18-24.

59. Havenaar R (2011) Intestinal health functions of colonic microbial metabolites: a review. Benef Microbes 2: 103-114.

60. Smith PM, Howitt MR, Panikov N, Michaud M, Gallini CA, et al. (2013) The microbial metabolites, short-chain fatty acids, regulate colonic Treg cell homeostasis. Science 341: 569-573. 